gastrointestinal bleeding. Hemospray achieved immediate and short term hemostasis in all cases. However, rebleed is possible in less than 48 hours after.

\section{IDDF2019-ABS-0329 EXPECTATION OF ASIA-PACIFIC PHYSICIANS AND PATIENTS TOWARDS IDEAL ERADICATION RATE OF ANTI-H. PYLORI REGIMENS}

${ }^{1}$ Yoen Young Chuah*, ${ }^{2}$ Deng Chyang Wu, ${ }^{3}$ Seng Kee Chuah, ${ }^{4}$ Jyh Chin Yang, ${ }^{5}$ Tzong Hsi Lee, ${ }^{6}$ Hong Zen Yeh, ${ }^{7}$ Chan Lin Chen, ${ }^{8}$ Yu Hwa Liu, ${ }^{9}$ Ping I Hsu. ${ }^{1}$ Ping Tung Christian Hospital, Taiwan; ${ }^{2}$ Kaohsiung Medical University Hospital, Taiwan; ${ }^{3}$ Kaohsiung Chang Gung Memorial Hospital, Taiwan; ${ }^{4}$ National Taiwan University Hospital, Taiwan; ${ }^{5}$ Far Eastern Memorial Hospital, Taiwan; ${ }^{6}$ Taichung Veterans General Hospital, Taiwan; ${ }^{7}$ Hualian Tzu Chi Medical Center, Taiwan; ${ }^{8}$ Shin Kong Wu Ho-Su Memorial Hospital, Taiwan; ${ }^{9}$ Kaohsiung Veterans General Hospital, Taiwan

\subsection{6/gutjnl-2019-IDDFabstracts.234}

Background The aims of the study were to survey (1) the ideal eradication rate for anti-H. pylori regimens from the expectation of Asia-Pacific physicians and patients (2) to investigate the expectation gaps of ideal eradication rate between physicians and patients.

Methods A questionnaire was disseminated to physicians who attended the Asia-Pacific Digestive Week 2015 meeting. In addition, a questionnaire was disseminated to $H$. pyloriinfected patients in Taiwan. Reported ideal eradication rates from the physicians and patients were collected and analyzed. Results A total of 754 physicians and 973 patients participated in the survey. The ideal eradication rate for anti-H. pylori regimens anticipated by Asia Pacific physicians was 91.5\%. Physicians from Malaysia had the highest expectation at 93.2\%. The expectation of physicians and patients towards ideal eradication rate of anti-H. pylori regimens were $91.1 \%$ and $93.1 \%$ respectively in Taiwan. The expectation on ideal eradication rate among patients was higher than that of physicians (93.1\% vs 91.1\%, $P<.001)$.

Conclusions It is time for Asia-Pacific physicians to implement more efficacious ideal anti- $H$. pylori regimens to meet the expectations of their patients and themselves.

\section{IDDF2019-ABS-0331 INTRALUMINAL BRACHYTHERAPY (ILBT) BOOST FOLLOWING CONCURRENT CHEMORADIATION IN PATIENTS OF INOPERABLE OESOPHAGEAL CANCER - A SINGLE INSTITUTE EXPERIENCE}

\begin{abstract}
'Diyya Khosla*, ${ }^{1}$ Rakesh Kapoor, 'Renu Madan, 'Sakshi Rana, 'Arun Oinam, 'Ranjit Singh, ${ }^{1}$ Narendra Kumar, ${ }^{1}$ Kannan Persamy, ${ }^{1}$ Budhi Yadav, ${ }^{2}$ Surinder Rana, ${ }^{3}$ Rajesh Gupta. ${ }^{1}$ Department of Radiotherapy and Oncology, Postgraduate Institute of Medical Education and Research (PGIMER), Chandigarh, India; ${ }^{2}$ Department of Gastroenterology, Postgraduate Institute of Medical Education and Research (PGIMER), Chandigarh, India; ${ }^{3}$ Department of Surgical Gastroenterology, Postgraduate Institute of Medical Education and Research (PGIMER), Chandigarh, India
\end{abstract}

\subsection{6/gutjnl-2019-IDDFabstracts.235}

Background Definitive concurrent chemoradiation is the standard of care for patients with inoperable oesophageal cancer. Intraluminal brachytherapy (ILBT) given as boost following concurrent chemoradiation increases the dose to tumor with sparing of adjacent normal structures. The aim of the present study was to analyse the response to concurrent

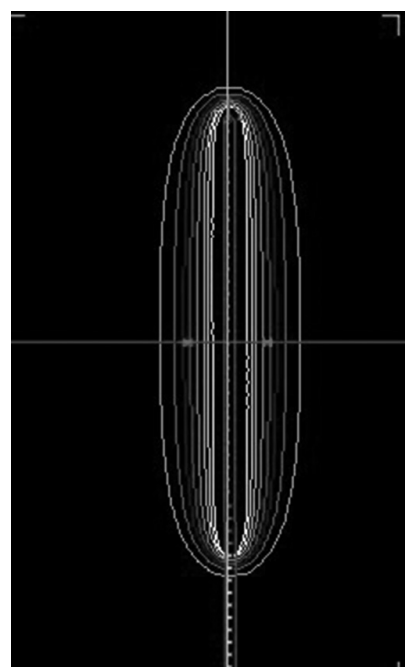

Abstract IDDF2019-ABS-0331 Figure 1 Isodose curves

chemoradiation followed by ILBT as a boost in inoperable oesophageal cancer patients.

Methods Twenty-two patients of carcinoma of the middle and lower oesophagus were included in the study. All patients received external beam radiotherapy of $40 \mathrm{~Gy}$ in 20 fractions over 4 weeks with concomitant chemotherapy with weekly cisplatin and 5-fluorouracil. Barium swallow and upper gastrointestinal endoscopy were performed for response assessment at 2 weeks post chemoradiation. The dose of brachytherapy was two fractions of 6 Gy delivered one week apart. Universal plastic bougie was inserted and a margin of $2 \mathrm{~cm}$ was given to residual tumor in superior and inferior direction. The dose was prescribed at $8 \mathrm{~mm}$ from midline and treatment was delivered by a high dose rate ${ }^{60}$ Co source (figure 1 ). Response evaluation was done at 1 and at 3 months after treatment completion.

Results The patient and tumor characteristics are shown in table 1 (table 1). The treatment was well tolerated and all patients completed the prescribed course of therapy. The swallowing ability improved in all patients except one patient after a month of therapy. No patient developed grade 3 or higher toxicity. Dysphagia score at one month follow up was grade I in 10 patients and grade 2 in 3 patients. None of the patients had grade 3 or 4 dysphagia after treatment. The median follow-up was 11 months. Three patients had progression of disease with worsening of dysphagia. Four patients had dysphagia due to stricture which was relieved by endoscopic dilatation.

Abstract IDDF2019-ABS-0331 Table 1 Patient and tumor characteristics $(n=22)$

\begin{tabular}{ll}
\hline Median age & 59 \\
Range & $42-70$ \\
\hline Gender & \\
Male & 14 \\
Female & 8 \\
Tumor length & \\
Mean & $7.05 \mathrm{~cm}$ \\
Range & $2.8-10.6 \mathrm{~cm}$ \\
Median number of chemotherapy cycles & 3 \\
\hline
\end{tabular}

